\title{
A study of the energetics of the $\mathrm{Cl}_{2} / \mathrm{MgO}(001)$ interface using correlation corrected periodic Hartree-Fock theory
}

\author{
Maureen I. McCarthy and Anthony C. Hess \\ Molecular Sciences Research Center, Pacific Northwest Laboratory, Richland, WA 99352 \\ N. M. Harrison and V. R. Saunders \\ SERC Daresbury Laboratory, Daresbury, Warrington WA4 4AD, United Kingdom
}

(Received 26 October 1992; accepted 3 December 1992)

\begin{abstract}
The energetics of the $\mathrm{Cl}_{2} / \mathrm{MgO}(001)$ interface were investigated using the $a b$ initio periodic Hartree-Fock (PHF) method and local density functional correlation corrections to PHF theory, as implemented in the program CRYSTAL92. Estimates of the correlation corrected PHF energies are made by post-SCF evaluations of three gradient corrected functionals. The correlation energy is calculated from the fully converged ground state PHF charge density and added to the PHF total energy. This is the first study of interfacial energetics using the correlation corrected PHF theory. PHF and correlation corrected molecule/surface binding energies are reported for seven orientations of the adsorbate with respect to the surface plane. Three of the configurations align the intramolecular axes along the surface normal and the remaining geometries arrange the molecules heat-to-tail, parallel to the surface plane. The most favorable interaction occurs when chlorine approaches a surface oxygen along the normal direction. This site preference is consistent with a classical electrostatic description of the physisorption process. The binding energy increases with decreasing surface coverage. At the most dilute coverage studicd (1:8) the PHF binding energy was $4.1 \mathrm{kcal} / \mathrm{mol}$ and the correlation corrected binding energies ranged from 9.2 to $10.3 \mathrm{kcal} / \mathrm{mol}$. All three functionals tended to increase the molecule/surface attractions, shorten the molecule/surface equilibrium distance, increase the curvature of the molecule/surface potential energy surface near equilibrium, and reduce the molecule/molecule repulsions.
\end{abstract}

\section{INTRODUCTION}

An investigation of the interfacial properties and energetics of $\mathrm{Cl}_{2}$ on $\mathrm{MgO}(001)$, using ab initio periodic Hartree-Fock (PHF) theory, was reported in an earlier work by $\mathrm{McC}$ arthy and Hess ${ }^{1}$ (hereafter referred to as MH1). That study reported results for two molecule/ surface orientations- $\mathrm{Cl}_{2}$ oriented normal to the surface approaching either a surface magnesium or oxygen site-as a function of surface coverage. The molecule was found to bind over the oxygen sites with a binding energy, $E_{\text {bind }}$, of $4.1 \mathrm{kcal} / \mathrm{mol}$ at a $1: 8$ coverage, but a coverage independent repulsive interaction was observed when $\mathrm{Cl}_{2}$ approached a magnesium site. Based on the computed physical properties of the $\mathrm{Cl}_{2} / \mathrm{MgO}(001)$ interface, these interactions were understood to be dominated by classical electrostatics. Very little charge redistribution (in either the adsorbate or the surface) occurred when $\mathrm{Cl}_{2}$ physisorbs onto $\mathrm{MgO}(001)$. Hence, at this level of theory, the process appears to be governed by electrostatic forces between the moments and polarizability of the molecule and the field and field gradients of the clean slab (MH1). The attractive molecule/surface interaction energy $\left(E_{\text {int }}\right)$ may be written as

$$
E_{\text {int }}=\mu_{z} F_{z}-\frac{1}{3} \Theta_{z z} \frac{d F_{z}}{d R}+\frac{1}{2} \alpha_{z z} F_{z}^{2} \ldots
$$

where, $\mu_{z}$ and $\Theta_{z z}$ are the normal components of the permanent dipole and quadrupole moments of the molecule, $\alpha$ is the molecular polarizability and $F_{z}$ is the normal com- ponent of the electric field. The interaction of $\mathrm{HCl}$, which contains a permanent dipole moment, with $\mathrm{MgO}(001)$ was also well described by the classical electrostatic model. ${ }^{2}$

The present work re-examines the energetics of the $\mathrm{Cl}_{2} / \mathrm{MgO}(001)$ interface using correlation corrected PHF theory which incorporates an estimate of the correlation energy into the calculations of the total energy of the system. This is accomplished through integration of three gradient corrected correlation functionals based on the selfconsistent PHF ground state charge density. A brief description of this method is given in Sec. II. The PHF and the correlation corrected energies for seven different $\mathrm{Cl}_{2}$ / $\mathrm{MgO}$ orientations are reported in Sec. III; these include the two configurations reported in $\mathrm{MH} 1$ and five additional adsorbate/surface geometries. The results are discussed in terms of the earlier study of $\mathrm{Cl}_{2}$ and on $\mathrm{MgO}(001)$ (MH1). Concluding remarks are made in Sec. IV.

\section{COMPUTATIONAL DETAILS}

The calculations in this study are done using the program CRYSTAL92, ${ }^{3,4}$ which is based on the methods of both PHF theory and local density functional correlation corrections to PHF theory. The fundamentals of PHF theory and its implementation in CRYSTAL92 have been discussed earlier. ${ }^{5,6}$ The method is well established and has been used to study a wide range of materials. ${ }^{1,6-11}$ The computational scheme initially calculates the ground state PHF charge densities at a given nuclear configuration. The contribution from the correlation functionals is then evaluated from this 
converged PHF charge density. Applications of correlation corrected PHF theory to bulk semi-conductors and semiionic compounds have been reported in works by Orlando et $a l^{9}$ and Harrison et al., ${ }^{12}$ respectively. The work of Harrison et al. ${ }^{12}$ found that gradient corrected correlation functionals yielded a reasonable description of the $\beta-\mathrm{MgCl}_{2}$ interlayer energetics. In the present work, we report results of calculations using three gradient corrected correlation functionals. Two are derived from studies of an electron gas and are referred to as $P 86^{13,14}$ and $P 91 .^{15}$ The third is determined from studies of the helium atom and is referred to as $\mathrm{CS}^{16,17}$ The results obtained using these three functionals in the current study of the $\mathrm{Cl}_{2} / \mathrm{MgO}$ interface were in qualitative agreement. Because these energetics have not been measured experimentally no attempt was made to compare the accuracy of the three functionals.

All the calculations in the present work were performed using a mixed split valence basis set of the form 8-51G on oxygen, 8-61G on magnesium ${ }^{1,18}$ and a standard Pople $6-21 \mathrm{G}^{*}$ on chlorine. The $\mathrm{Cl}_{2}$ geometry was fixed at the calculated $\left(6-21 \mathrm{G}^{*}\right)$ equilibrium distance, $1.9926 \AA$. The correlation corrected equilibrium distances were all within $3 \%$ of this value. Essentially no change in the intramolecular distance was observed due to interactions between the molecule and the surface. The model $\mathrm{Cl}_{2} / \mathrm{MgO}$ system contains two molecules per unit cell (one "above" and one "below" the slab) on either side of the twodimensional three layer slab of $\mathrm{MgO}$ (generating a mirror plane). The total energies are calculated with the orientations of the adsorbates fixed with respect to the surface plane. The slab thickness (three layers) was chosen such that the charge density of the center atoms resembled those found in bulk $\mathrm{MgO}$ (MH1). No interaction was observed between the top and bottom molecules and calculations with and without the mirror plane [two or one molecule(s) per unit cell] gave the same binding energies (MH1). The lattice constants $a$ and $b$ of the slab (along the $x$ and $y$ directions, respectively) are set such that the $\mathrm{MgO}$ supercell defines a molecule/molecule spacing at the desired coverage. The tolerance parameters used to evaluate the Coulomb and exchange series in all of these calculations were $\left(s_{c}=t_{m}=5\right)$ and $\left(s_{\mathrm{ex}}=p_{\mathrm{ex}}^{g}=5\right.$ and $\left.p_{\mathrm{ex}}^{1}=10\right) .{ }^{5}$

\section{RESULTS AND DISCUSSION}

The binding energy, $E_{\text {bind }}$, of $\mathrm{Cl}_{2}$ on $\mathrm{MgO}(001)$ is defined as the energy required to remove one molecule from the surface, at a given coverage,

$$
E_{\text {bind }}=\frac{1}{2}\left[E_{n \mathrm{MgO}}+E_{2 \mathrm{Cl}_{2}}-E_{n \mathrm{MgO} / 2 \mathrm{Cl}_{2}}\right] \text {. }
$$

The terms on the right hand side of (2) represent the total energies per unit cell of, the clean (001) slab ( $n$ denotes the number of $\mathrm{MgO}$ formula units in the supercell), the isolated chlorine molecules and the total system at the equilibrium configuration. The reported coverages, 1:2, 1:4 and $1: 8$, imply that $50 \%, 25 \%$ or $12.5 \%$ of like sites on the surface contain chlorine molecules. The PHF and the correlated corrected binding energies for seven different molecule/surface orientations are reported in Table I. The surface coverages are expressed in terms of the molecule/ molecule spacings (square lattice for normal orientations and a rectangular net for parallel configurations). A schematic representation of these configurations is shown in Fig. 1. In three of these geometries the molecules were perpendicular to the $\mathrm{MgO}(001)$ face over either an oxygen [Fig. 1(a)] or surface magnesium atom [Fig. 1(b)] or over the center of an $\mathrm{Mg} / \mathrm{O}$ subcell [Fig. 1(c)]. The other four geometries fixed the molecules parallel to the surface oriented either between two oxygens [Fig. 1(d)] or between two magnesiums [Fig. 1(e)] or straddling either an oxygen [Fig. 1(f)] or magnesium atom [Fig. 1(g)] (aligned along a diagonal line of like surface sites). The latter geometries align the molecules head-to-tail in a rectangular lattice on the surface.

The most favorable configuration (of the seven studied) occurs when the $\mathrm{Cl}_{2}$ approaches normal to a surface oxygen. The physical properties and PHF energetics of this molecule/surface orientation were discussed in detail in MH1. It is evident from the data in Table I that the PHF and the correlation corrected binding energies increase with decreasing surface coverage. This effect is a direct result of the purely repulsive interactions between molecules on the surface which cause the binding energy to increase as the surface coverage decreases. Hence, adsorbates in this configuration are predicted to "wet" the surface. The largest binding energy occurs at the most dilute coverage $(1: 8)$ and the correlation corrected values range from $9.2 \mathrm{kcal} / \mathrm{mol}$ to $10.3 \mathrm{kcal} / \mathrm{mol}$. The analogous configuration with the molecule approaching a surface magnesium shows a purely repulsive interaction at all coverages.

The interfacial energetics observed in the present work are consistent with (and strengthen) the arguments put forward in the earlier paper (MH1) regarding the nature of the $\mathrm{Cl}_{2} / \mathrm{MgO}$ interaction. The physical properties reported in MH1 (band structure, total and projected density of states, charge density and density difference maps, Mulliken analyses, and electrostatic potential electric field maps) indicated that the physisorption of $\mathrm{Cl}_{2}$ onto $\mathrm{MgO}(001)$ did not substantially alter the charge density of either object. From these observations and from the calculated maps of the electrostatic potential and electric fields, it was deduced that the attractive interaction could be accounted for by the classical electrostatic expression, (1). This expansion (1) is dominated by the term containing the normal components of the molecular quadrupole moment and the field gradient. The magnitude and sign of the latter term dictated favorable binding of the adsorbate over an oxygen with its quadrupole moment aligned along the $z$ component of the electric field (intramolecular axis perpendicular to the surface plane). The present work considered five additional adsorbate/surface orientations, but the former configuration remained the most energetically favorable. The site preference is maintained (probably through higher order terms) even when the quadrupole moment/field gradient interaction is reduced as the molecule is tipped off the surface normal.

The three correlation functionals display the same relative behavior for all of the geometries studied. Three main 
TABLE I. Energetics of the $\mathrm{Cl}_{2} / \mathrm{MgO}$ interface at different molecule/surface orientations and molecule/molecule spacings (surface coverages). The molecule/surface interaction energies reported are from PHF calculations and calculations using three different estimates of the correlated corrected energies. The equilibrium distance of the molecule from the surface is expressed as $Z_{\mathrm{Cl} / s}$ Positive values indicate an energetically favorable interaction. [ 1 denotes orientations with the molecule normal to the surface plane and $\|$ indicates configurations with the molecule parallel to the surface plane.]

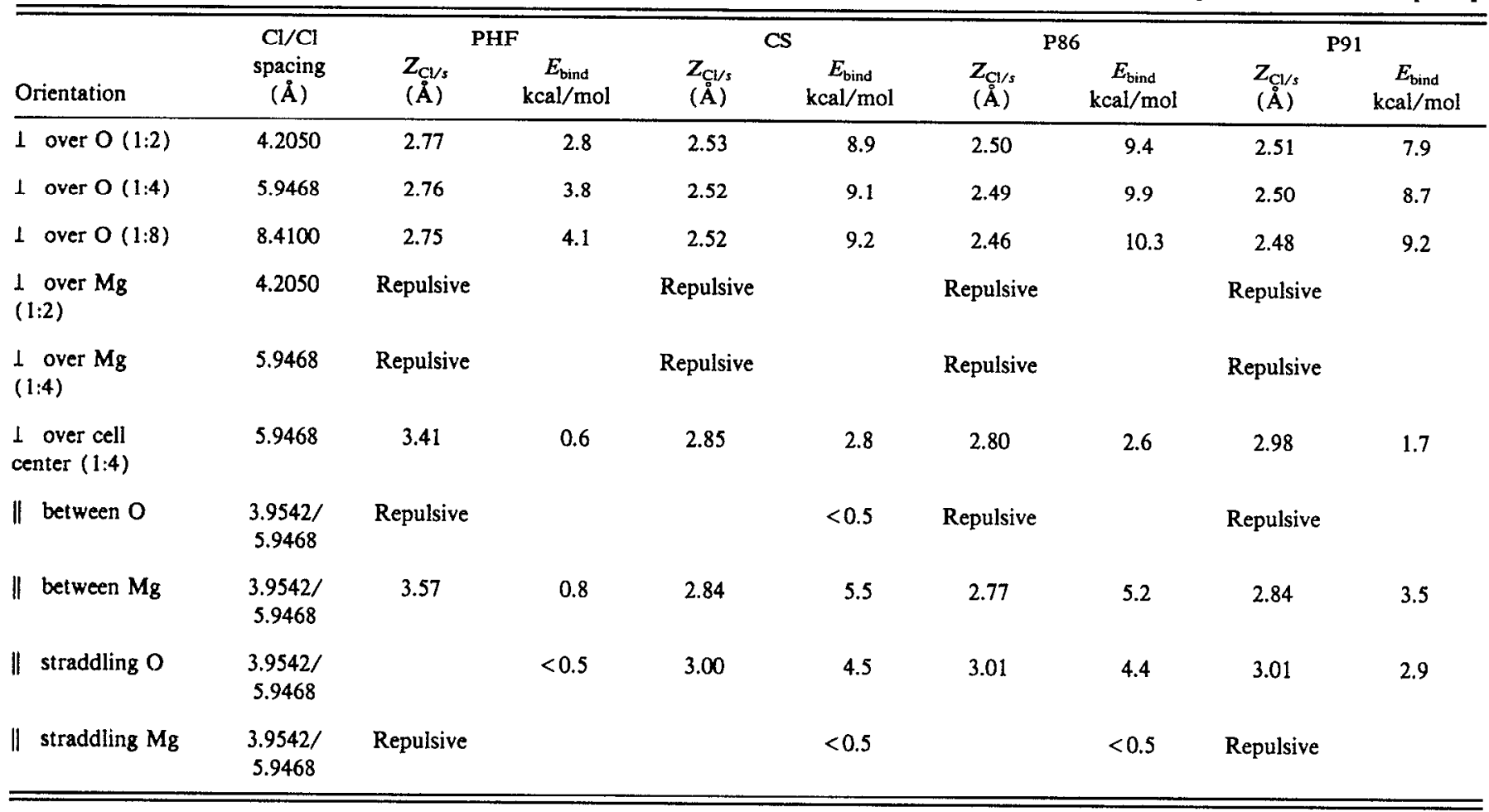

effects were observed in the correlated energetics relative to the PHF results: (1) a substantial increase in the molecule/surface binding energy; (2) a shift in the molecule/surface equilibrium geometry to shorter dis- tances; and (3) an increase in the curvature of the molecule/surface potential energy surface. Binding energies calculated using the three functionals are in qualitative agreement with each other (within 1-2 kcal/mol) at all
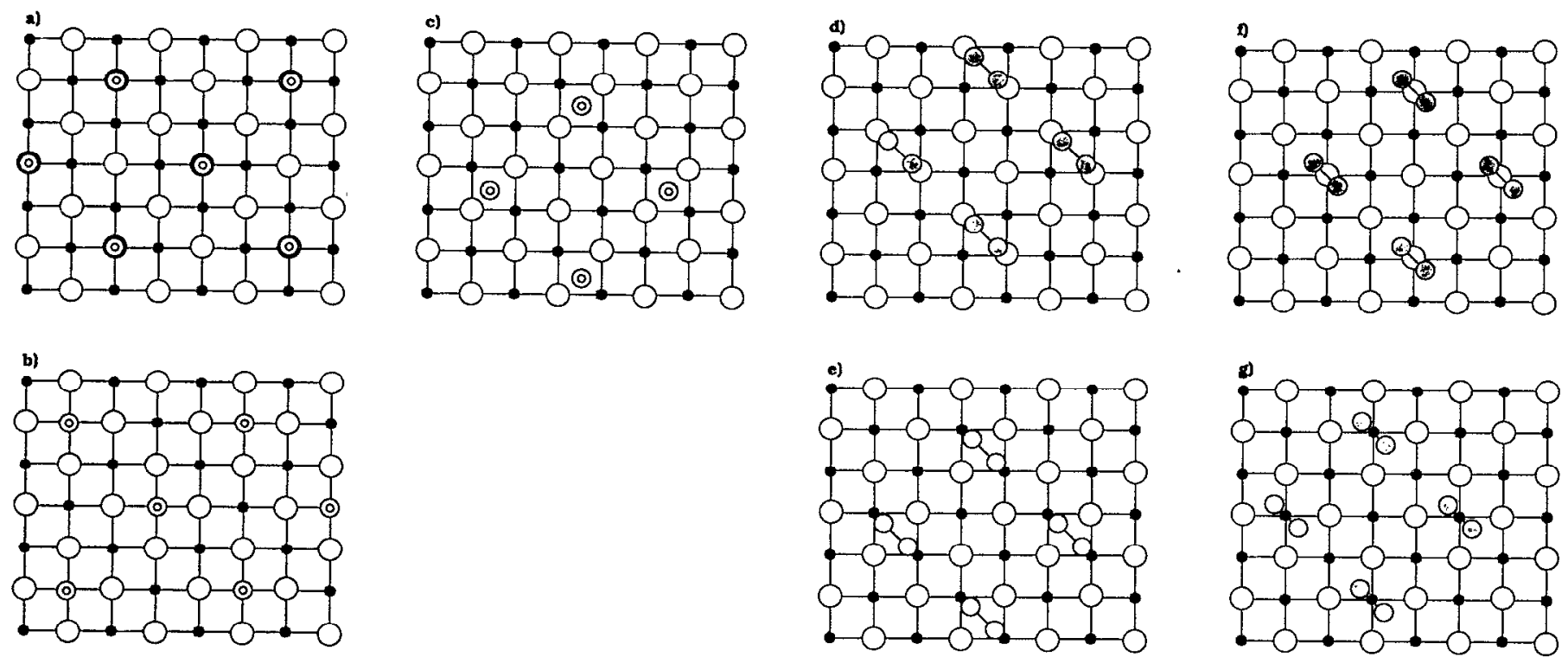

FIG. 1. Orientations of $\mathrm{Cl}_{2}$ on $\mathrm{MgO}(001)$ projected onto the ab plane (along $x$ and $y$ ) of the slab; (a)-(c) molecules are aligned along the surface normal over an oxygen (a), magnesium (b), or a cell center (c) site, (d) $-(\mathrm{g})$ molecules are arranged head-to-tail parallel to the surface plane, between two oxygens (d), between two magnesiums (e), straddling an oxygen (f) or straddling a magnesium ( $g$ ) [open circles are oxygens, filled circles are magnesiums and shaded circles are chlorines] 


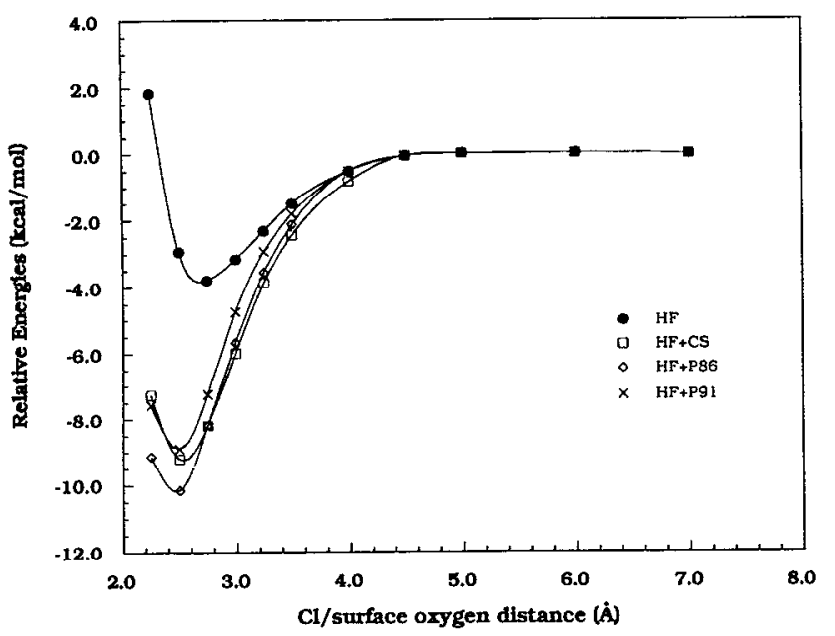

FIG. 2. Interaction of $\mathrm{Cl}_{2}$ on $\mathrm{MgO}(001)$ with the molecules aligned along the surface normal over a surface oxygen at a 1:8 coverage (molecule/molecule spacing $=8.41 \AA$ ). Relative energetics are shown for the PHF and correlated corrected PHF results. The asymptotic limit in all curves has been set to zero to eliminate the offset in the correlated corrected energies.

configurations studied. The inclusion of correlation increases the binding energies by $1-7 \mathrm{kcal} / \mathrm{mol}$ and decreases the equilibrium molecule/surface bond distance by $\sim 0.2$ $\AA$. Figure 2 shows the total PHF and correlated corrected energies for the most favorable $\mathrm{Cl}_{2} / \mathrm{MgO}$ orientation (1:8, $\mathrm{Cl}_{2}$ normal to surface oxygen). All of the curves have been zeroed to the asymptotic value to remove the offsets in the energies calculated from the correlation functionals. Table II shows the variation of the correlation energies upon dilation of periodic arrays of chlorine molecules. Inclusion of correlation serves to reduce the short range intermolecular repulsion at molecule/molecule separations corresponding to the 1:1 and 1:2 coverages. However, the functionals do not affect the energetics of the 1:4 and 1:8

TABLE II. Interaction energies of the periodic net of $\mathrm{Cl}_{2}$ molecules (per molecule) calculated at the spacings corresponding to the surface coverages reported in Table I. In the first four entries the intramolecular axes are aligned parallel to each other, corresponding to an approach to the (001) face of $\mathrm{MgO}$ along the surface normal. The final entry describes the interaction between molecules aligned head-to-tail in the configuration corresponding to adsorbates arranged parallel to the $(001)$ surface plane. Positive values indicate a repulsive interaction.

\begin{tabular}{|c|c|c|c|c|c|}
\hline Geometry & $\begin{array}{c}\text { Net spacing } \\
(\tilde{A})\end{array}$ & $\begin{array}{c}\text { PHF } \\
\mathrm{kcal} / \mathrm{mol}\end{array}$ & $\begin{array}{c}\mathrm{CS} \\
\mathrm{kcal} / \mathrm{mol}\end{array}$ & $\begin{array}{c}\mathrm{P} 86 \\
\mathrm{kcal} / \mathrm{mol}\end{array}$ & $\begin{array}{c}\mathrm{P91} \\
\mathrm{kcal} / \mathrm{mol}\end{array}$ \\
\hline$\| 1: 1$ & 2.9730 & +34.0 & +19.9 & +17.1 & +19.4 \\
\hline$\| \quad 1: 2$ & 4.2050 & +0.8 & -0.4 & +0.1 & +0.4 \\
\hline$\| 1: 4$ & 5.9468 & +0.1 & +0.1 & +0.1 & +0.1 \\
\hline || $1: 8$ & 8.4100 & +0.0 & +0.0 & +0.0 & +0.0 \\
\hline $\begin{array}{l}\text { Head-to-tail } \\
\text { rectangle }\end{array}$ & $3.9547 / 5.9468$ & +0.4 & +0.2 & +0.3 & +0.4 \\
\hline
\end{tabular}

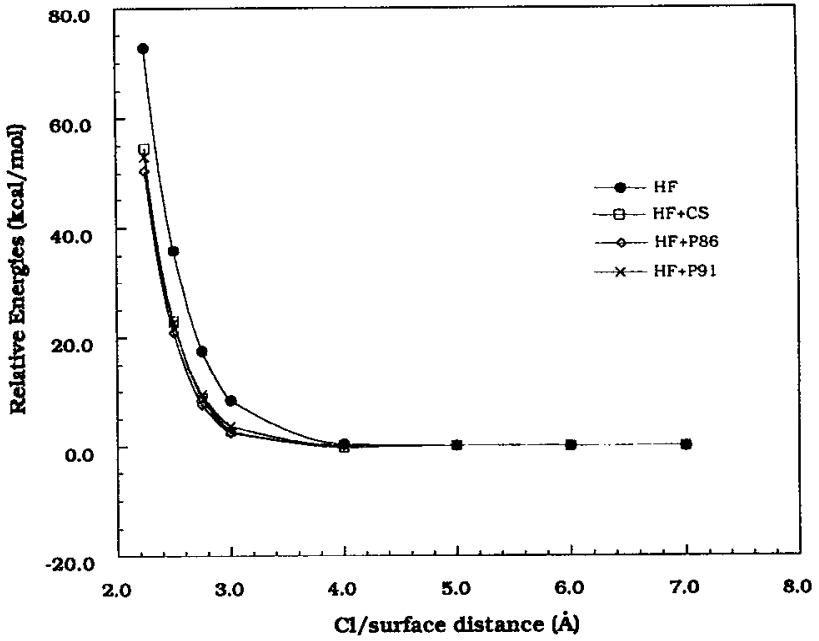

FIG. 3. Interaction of $\mathrm{Cl}_{2}$ on $\mathrm{MgO}(001)$ with the molecule arranged head-to-tail on the surface between two oxygens. Relative energetics are shown for the PHF and correlated corrected PHF results. The asymptotic limit in all curves has been set to zero to eliminate the offset in the correlated corrected energies.

spacings, indicating that the correlation interaction is comparatively short ranged. This behavior is expected from the local nature of the functionals. The decrease in the molecule/molecule repulsion energy at the 1:2 spacing accounts in part for the greater spread in binding energies between the PHF and correlation corrected data at that coverage. At the three other bound configurations (perpendicular over the cell center, parallel between magnesiums and parallel straddling an oxygen) the binding energies at the PHF level are all $\leqslant 1.0 \mathrm{kcal} / \mathrm{mol}$ while the correlation corrected values range from 1.7 to $5.5 \mathrm{kcal} / \mathrm{mol}$. The three remaining configurations display purely repulsive adsorbate/surface interactions at both the PHF and correlated levels of theory (see Table I). An example of this behavior is evident in Fig. 3 which shows the total energy data for the configuration with the molecules approaching the surface parallel to the $(001)$ face between two oxygens.

The present data corroborates earlier observations that the correlation functionals give rise to significant corrections to short-range forces. Long-range effects, such as dispersion forces, which are proportional to $r^{-6}$, are not explicitly treated in either the PHF or the correlated corrected PHF theories. This implies that the potential energy curves for the molecules approaching the surface, obtained from the correlation corrected PHF calculations, do not accurately account for the behavior at large molecule/surface separations. It appears that the functionals do correctly describe the gradient of the energy near the minima (and hence the lattice constants ${ }^{12}$ ) and the energetics of the asymptote. The binding energetics may, therefore, be well described even though the shape of the potential energy curve is in error; in the absence of empirical data on this system it is impossible to quantitatively assess these functionals. 


\section{CONCLUSIONS}

We have investigated the $\mathrm{Cl}_{2} / \mathrm{MgO}(001)$ interface using PHF theory and three density functional correlation corrections. Total energies were reported for chlorine approaching the surface in seven adsorbate/surface orientations. The energetically most favorable configuration occurs when the intramolecular axes of the diatomics are aligned along the surface normal, over an oxygen atom. This is consistent with the arguments presented in the earlier study (MH1). The molecule/surface binding energies were found to increase with decreasing surface coverage. At the most dilute coverage studied (1:8) the PHF binding energy was $4.1 \mathrm{kcal} / \mathrm{mol}$ and the correlated corrected energies were 9.2 to $10.3 \mathrm{kcal} / \mathrm{mol}$. In general, the correlation functionals increased the molecule/surface binding energies (by 1-7 kcal/mol) and decreased the molecule/ molecule repulsions. The energetics calculated from the three functionals showed qualitatively similar behavior for all molecule/surface geometries. They increased the adsorbate/surface binding energies, decreased the adsorbate/surface equilibrium distances and increased the curvature in the potential energy surface in the vicinity of the equilibrium position. Experimental data is required to assess the relative merits of the functionals.

\section{ACKNOWLEDGMENTS}

The authors wish to thank Mario Causa and Eduardo Apra for helpful discussions. This work was supported by the Division of Chemical Sciences Office of Basic Energy Sciences, U. S. Department of Energy under Contract No. DE-AC06-76 RLO 1830 with Pacific Northwest Labora- tory and the Advanced Industrial Concepts Division of the Office of Conservation and Renewable Energies (Contract No. 16697). We also wish to thank the Scientific Computing Staff, Office of Energy Research, U. S. Department of Energy for a grant of computing time at the National Energy Research Supercomputer Center.

${ }^{1}$ M. I. McCarthy and A. C. Hess, J. Chem. Phys. 96, 6010 (1992).

${ }^{2}$ M. I. McCarthy and A. C. Hess, J. Chem. Phys. (submitted).

${ }^{3}$ R. Dovesi, V. R. Saunders, and C. Roetti, CRYSTAL92 (manual available upon request from V. R. Saunders, Daresbury, England, 1992).

${ }^{4}$ R. Dovesi, C. Pisani, C. Roetii, and V. R. Saunders, Program CRYs TAL92 (Quantum Chemistry Program Exchange, Indiana University, Department of Chemistry, Bloomington, Indiana, 1988).

${ }^{5}$ C. Pisani, R. Dovesi, and C. Roetti, Hartree-Fock Ab Initio Treatment of Crystalline Systems (Springer-Verlag, New York, 1988).

${ }^{6}$ A. C. Hess and V. R. Saunders, J. Phys. Chem. 96, 4367 (1992).

${ }^{7}$ A. C. Hess and J. C. White, J. Phys. Chem. (submitted).

${ }^{8}$ R. Nada, C. R. A. Catlow, and R. Dovesi, Phys. Chem. Min. 17, 353 (1990).

${ }^{9}$ R. Orlando, R. Dovesi, C. Roetti, and V. R. Saunders, J. Phys. C 2, 7769 (1990).

${ }^{10}$ L. Salasco, R. Dovesi, R. Orlando, and M. Causa, Mol. Phys. 72, 267 (1991).

"B. Silvi, Theochem. 72, 129 (1991).

${ }^{12}$ N. M. Harrison, V. R. Saunders, E. Apra, M. Causa, and R. Dovesi, J. Phys. 4, 2261 (1992).

${ }^{13}$ J. P. Perdew, Phys. Rev. B 33, 8822 (1986).

${ }^{14}$ J. P. Perdew, Phys. Rev. B 34, 7406 (1986).

${ }^{15}$ J. P. Perdew, in Electronic Structure of Solids '91, edited by P. Ziesche and J. Eschrig (Akademie Verlag, Berlin, 1991); J. P. Perdew, J. A. Chevary, S. H. Vosko, K. A. Jackson, M. R. Pederson, D. Singh, and C. Fiolhais, Phys. B 46, 6671 (1992).

${ }^{16} \mathrm{R}$. Colle and D. Salvetti, Theor. Chim. Acta. 37, 329 (1975).

${ }^{17}$ C. Lee, W. Yang, and R. G. Parr, Phys. Rev. B 37, 785 (1988).

${ }^{18}$ M. Causa, R. Dovesi, C. Pisani, and C. Roetti, Phys. Rev. B 33, 1308 (1986). 